\title{
BMJ Factors associated with breastfeeding Open in England: an analysis by primary care trust
}

\author{
Laura L Oakley, ${ }^{1}$ Mary J Renfrew, ${ }^{2}$ Jennifer J Kurinczuk, ${ }^{1}$ Maria A Quigley ${ }^{1}$
}

To cite: Oakley LL, Renfrew MJ, Kurinczuk JJ, et al. Factors associated with breastfeeding in England: an analysis by primary care trust. BMJ Open 2013;3: e002765. doi:10.1136/ bmjopen-2013-002765

- Prepublication history and additional material for this paper is available online. To view these files please visit the journal online (http://dx.doi.org/10.1136/ bmjopen-2013-002765)

Received 20 February 2013 Revised 17 April 2013 Accepted 26 April 2013

This final article is available for use under the terms of the Creative Commons Attribution Non-Commercial 2.0 Licence; see http://bmjopen.bmj.com

${ }^{1}$ Policy Research Unit in Maternal Health and Care, National Perinatal

Epidemiology Unit, University of Oxford, Oxford, UK

${ }^{2}$ Mother and Infant Research Unit, College of Medicine, Dentistry and Nursing, University of Dundee, Dundee, UK

\section{Correspondence to} Dr Laura Oakley; laura.oakley@npeu.ox.ac.uk

\section{ABSTRACT}

Objectives: To identify the sociodemographic factors associated with variation in area-based breastfeeding in England; to calculate the predicted breastfeeding rates adjusted for sociodemographic variations.

Design: Ecological analysis of routine data using random effects logistic regression.

Setting: All 151 primary care trusts (PCTs) in England 2010-2011.

Outcome measures: PCT level data on breastfeeding: initiation, any and exclusive breastfeeding at 6-8 weeks.

Results: There was considerable variation in breastfeeding across PCTs (breastfeeding initiation mean $72 \%$, range $39-93 \%$; any breastfeeding at 6-8 weeks mean $45 \%$, range $19-83 \%$; exclusive breastfeeding at 6-8 weeks mean $32 \%$, range $14-58 \%$ ), with London PCTs reporting markedly higher rates. Maternal age was strongly associated with area-based breastfeeding, with a $4-6 \%$ increase in odds of breastfeeding associated with a unit increase in the percentage of older mothers. Outside London, the proportion of the local population from a Black and Minority Ethnic (BME) background, compared with those from a White British background, was associated with higher breastfeeding (1-3\% increase in odds per unit increase in the proportion from a BME background). Area-based deprivation was associated with reduced odds of breastfeeding (21-32\% reduced odds comparing most deprived quintile to least deprived quintile). Weaker associations were observed between sociodemographic factors and breastfeeding in London PCTs. Very few PCTs reported breastfeeding figures substantially above or below the national average, having adjusted for variations in sociodemographic factors.

Conclusions: Our results show striking associations between sociodemographic factors and breastfeeding at the area level, with much of the variation in breastfeeding rates explained by the sociodemographic profile. The sociodemographic context of breastfeeding is clearly important at the area level as well as the individual level. Our findings can be used to inform decision-making relating to local priorities and service provision.

\section{INTRODUCTION}

Breastfeeding confers multiple benefits on both infants and mothers, with evidence linking breastfeeding to a lower risk of many

\section{ARTICLE SUMMARY}

Article focus

- The primary aim of the reported study was to identify the sociodemographic factors independently associated with variation in area-based breastfeeding in England (breastfeeding initiation, any and exclusive breastfeeding rates at $6-8$ weeks).

- The secondary aim was to calculate the predicted area-based breastfeeding rates adjusted for sociodemographic variations using multivariable modelling.

Key messages

- Striking associations between sociodemographic factors and breastfeeding at the area level explain much of the variation in breastfeeding rates between areas. These associations were strongest in PCTs outside London; for London PCTs, the associations were less consistent.

- After adjustment for sociodemographic factors, most PCTs have breastfeeding rates in line with those expected given the overall trends; however, the breastfeeding rates are still comparatively low, especially for exclusive breastfeeding.

- The findings of this study confirm the importance of the sociodemographic context and support the view that breastfeeding interventions need to be tailored to the needs of a particular setting. Our results can be used to compare breastfeeding across areas with similar sociodemographic characteristics and to inform service commissioning.

Strengths and limitations of this study

- To our knowledge, this is the first UK study to investigate the relationship between sociodemographic factors and breastfeeding at an areabased level. We used routine data to look at breastfeeding prevalence and sociodemographic factors, and our analysis covers all English PCTs.

- This is an ecological study, and as such our results are subject to the usual limitation that causality cannot be inferred. It is possible that variations in breastfeeding rates may be partially explained by area-level factors not measured in this study.

- The small sample number of PCTs in London may affect our confidence in the results from our analysis of London PCTs. 
adverse outcomes ${ }^{12}$ including gastroenteritis, ${ }^{3}{ }^{4}$ respiratory disease, ${ }^{4}{ }^{5}$ necrotising enterocolitis ${ }^{6}$ and otitis media ${ }^{15}$ in infants, and a lower risk of breast cancer in mothers. ${ }^{1} 27$ Breastfeeding has also been linked to other health, social and cognitive outcomes including childhood obesity and cognitive development. ${ }^{8} 9$

Current UK guidance recommends that infants are exclusively breastfed to 6 months of age. ${ }^{10}$ In England, just over four of five $(83 \%)$ mothers now start breastfeeding, ${ }^{11}$ but the recent improvements in initiation have not been reflected to the same extent in duration and exclusivity; by 6 weeks, the proportion breastfeeding has dropped to $57 \%$. Only $36 \%$ of mothers are still breastfeeding at 6 months. Twenty-four per cent of mothers are breastfeeding exclusively at 6 weeks, and there are just $13 \%$ at 4 months. ${ }^{11}$ Percentages are lower still in the other constituent countries of the UK, and by international comparisons, UK breastfeeding rates compare poorly with those of other European countries. ${ }^{12}$

A recent report estimated that a moderate increase in breastfeeding rates in the UK could save over $£ 17$ million a year as a result of reduced costs for treating four acute infant diseases, with further savings accruing from the resulting reduction in breast cancer cases. ${ }^{2}$ In the UK, breastfeeding is a major factor in inequalities in health; not being breastfed is both a cause and a consequence of social inequalities. ${ }^{2}$ Improving breastfeeding rates in the UK has been a key focus of successive governments over the last decade, ${ }^{13}{ }^{14}$ with the recent public health outcomes framework for England identifying breastfeeding as a key indicator for health improvement. ${ }^{15}$

Previous studies have identified a variety of sociodemographic and behavioural factors, including area of residence, maternal age, socioeconomic background, maternal education, ethnicity, smoking behaviour and maternal obesity, as being associated with breastfeeding in both the UK and other high income countries. ${ }^{11} 16-28$ However, these are based on the analysis of individual women and little is known about the factors that are associated with breastfeeding at the area level. In England, breastfeeding data have formed part of the Department of Health (DH) Vital Signs Monitoring Return since 2004 and are routinely reported at a number of different aggregate levels. Studies based on area-level data are well placed to make use of routinely collected data such as this, and can help to inform commissioning of services as well as providing a framework with which to evaluate relevant interventions. A recent study by Freemantle et $a l^{29}$ used an approach similar to the one described here to look at factors associated with primary care trust (PCT) level perinatal and infant mortality.

The aim of this study was to identify the sociodemographic factors associated with variation in area-based breastfeeding rates in England.

\section{METHODS}

We conducted an area-based analysis making use of data routinely collected at the PCT level. Until their abolition in April 2013, PCTs were the administrative bodies responsible for commissioning all primary, community and secondary health services in a defined geographical area in England. For the time period under study, PCTs ranged in population size and annual number of births from 1134 to 14972 births (mean 4550, SD 2429; median 3823, IQR 2952-5591; 2010 data). All 151 PCTs in England (boundaries as of 2010) were eligible for inclusion. Ethical approval was not required as the dataset comprised publicly available routine data at the aggregate level.

\section{Outcome measures}

In England, the breastfeeding status at birth and at $6-8$ weeks is routinely collected shortly after birth and at the 6-8 week infant review. We focused on three breastfeeding outcomes: breastfeeding initiation at birth, any breastfeeding at $6-8$ weeks, and total (exclusive) breastfeeding at $6-8$ weeks. ${ }^{30}$

Breastfeeding is recorded as being initiated if infants receive any breast milk in the first $24 \mathrm{~h}$ after birth. At 6-8 weeks, infants are classified into one of three categories according to feeding method in the preceding 24 h: not breastfed; partially breastfed; or totally breastfed (hereafter referred to as 'exclusively' breastfed). The last two groups are combined to give the outcome 'any breastfeeding'. Data on breastfeeding outcomes at these two time points (birth and 6-8 weeks) are released quarterly by DH. For this analysis, the overall figures for 2010-2011 were calculated by summing the raw quarterly actual data. PCTs were included where reported data for at least two of the four quarters of 2010-2011 met DH data coverage standards $(\geq 95 \%$ data coverage for initiation; for $6-8$ week data, $\geq 90 \%$ and $\geq 95 \%$ data coverage for quarters 1-3 and quarter 4, respectively) and passed validation checks (relating to consistency in the reporting of the number of maternities/infants due in a 6-8 week check). According to the usual DH practice, infants for whom a breastfeeding status (initiation or at $6-8$ weeks) was not recorded were considered to be not breastfed, as long as the proportion of infants falling in this category within an individual PCT was small $(<5 \%$ or $<10 \%$ depending on the threshold for the quarter).

\section{Explanatory variables}

The following area-based sociodemographic indicators were included in our analysis: area-based deprivation, the proportion of births to older (aged $>35$ years) and younger (aged $<20$ years) mothers, and the proportion of the PCT population deriving from Black and Minority Ethnic (BME) backgrounds. We included the prevalence of maternal smoking as an additional explanatory factor.

We used the 2010 Index of Multiple Deprivation (IMD) as our indicator of material deprivation. ${ }^{31}$ Data 
on 38 domains contribute to this index and are combined to reflect a broad concept of deprivation. IMD is calculated at the level of 'lower super output area' (LSOA), of which there are 32482 in England. The score for each PCT is the average of the constituent LSOAs.

The estimated proportion of each PCT population from a BME background was derived from the Office for National Statistics (ONS) Population Estimates by Ethnic Group (PEEG) for 2009.32 PEEG is calculated using a cohort component methodology using data from the 2001 Census and recent data on births, deaths and migration. BME was defined as non-White British.

The percentage of women smoking at delivery by PCT is reported quarterly by PCTs in England. In this analysis, we used the figures for 2010-2011 published by DH for England. ${ }^{30}$ As with breastfeeding data, DH imposes quality checks on these data (minimum of $\geq 95 \%$ data coverage, reported numbers of maternities and women smoking/not smoking must satisfy consistency checks).

Information on the percentage of births at the PCT level occurring to older mothers (women aged older than 35) and younger mothers (women aged less than 20) in 2010-2011 was derived from Hospital Episode Statistics (HES) data reported by the Child and Maternal Health Observatory. ${ }^{33}$

Data on other factors strongly associated with breastfeeding, for example, maternal education, are not collected routinely at the PCT level. We were therefore unable to include other factors of interest in the analysis.

\section{Statistical analysis}

The association between sociodemographic variables and all three breastfeeding outcomes was investigated using separate logistic regression models. Random effects logistic regression models were used to take into account the clustered hierarchical nature of the data.

Most explanatory variables were analysed as continuous variables, for example, the proportion of births in a PCT to mothers aged less than 20; thus, we estimated the effect of a one percentage point increase in each variable on the breastfeeding proportion in the PCT. IMD scores were divided into quintiles for ease of analysis.

Preliminary analyses of the data revealed a striking difference in the sociodemographic profile of London PCTs when compared with PCTs outside London. There was also evidence that the effect of area-based deprivation differed according to whether PCTs were in London or not. For this reason, all analysis was stratified by region (London vs non-London).

Variables (or any resulting ORs for that variable) which were associated ( $p<0.10$ using Wald's test for at least one relevant OR) with breastfeeding in univariable analysis were included in multivariable random effects logistic regression models. The final model included all variables which were associated $(p<0.05$ using Wald's test for at least one relevant OR) with the outcome after adjusting for other factors in the model. This strategy was repeated for each relationship under study.

The final multivariable models were used to generate predicted proportions of all breastfeeding outcomes for PCTs in England, assuming fixed effects for the explanatory variables shown in the tables. Differences between observed and predicted proportions were examined by calculating standardised residuals for all PCTS; those with observed proportions that were two or more standardised residuals above or below the predicted proportions were highlighted as possible outliers. These figures can be used to provide a more suitable comparison of local performance, as they take into account the distribution of sociodemographic factors that we know affect breastfeeding.

All statistical analyses were conducted using Stata V.11 (StataCorp, College Station, Texas, USA); all tests were two tailed and a 5\% significance level was used unless specified otherwise.

\section{RESULTS}

All 151 PCTs in England in 2010 were included for the analysis of breastfeeding initiation, but 10 PCTs failed to report 6-8 week data that met DH quality controls for two or more quarters. Therefore, information on breastfeeding initiation was available for 151 PCTs, and for breastfeeding status at $6-8$ weeks, data were available for 141 PCTs.

Breastfeeding initiation varied across the PCTs from $39 \%$ to $93 \%$, with a mean of $72 \%$ (table 1). For breastfeeding status at 6-8 weeks, the mean percentage of any breastfeeding was $45 \%$ (range 19-83\%) and for exclusive breastfeeding, 32\% (range 14-58\%). On average, one in five $(19 \%)$ births in each PCT were to women aged over 35 (range 9-42\%) and 6\% were to women aged under 20 (range 1-12\%). The mean proportion of mothers who were smoking at the time of delivery was $15 \%$ (range 3-33\%). The proportion of the PCT population from a BME background averaged $19 \%$ across all PCTs (range 4-67\%).

The profile of London PCTs differed markedly from PCTs in the rest of England. Breastfeeding tended to be more common in London PCTs, with average breastfeeding initiation at $86 \%$, compared with $69 \%$ for PCTs outside London. Equivalent figures for any and exclusive breastfeeding at 6-8 weeks in London were $68 \%$ and $41 \%$, and outside London, $40 \%$ and $29 \%$. London PCTs also had a higher proportion of births to older mothers (25\% vs $18 \%$ ), a higher proportion of residents from a BME background ( $40 \%$ vs $13 \%$ ), a lower proportion of births to teenage mothers (3\% vs $7 \%$ ) and a lower prevalence of maternal smoking at delivery $(7 \%$ vs $17 \%)$. The deprivation profile was similar when comparing London and non-London PCTs. All further results are shown separately for PCTs outside $(\mathrm{n}=120)$ and inside $(\mathrm{n}=31)$ London. 
Table 1 Distribution of the prevalence of breastfeeding and socio-demographic variables across PCTs

\section{All PCTs $(n=151)$}

Median (IQR)

Minimum, maximum

\section{Breastfeeding}

Breastfeeding initiation $72.3(11.2) 72.8$ (65.2-79.9) $39.0,92.9$

$(\%)$

Any breastfeeding at 6-8 45.3 (15.0) 42.3 (34.5-54.0) $19.2,83.1$

weeks (\%)*

Exclusive breastfeeding $31.6(9.1) \quad 31.4(24.9-37.1) \quad 14.2,58.2$

at $6-8$ weeks $(\%)^{*}$

Sociodemographic

IMD (raw score)

Mothers aged 35+ (\%)

Mothers aged $<20(\%)$

Population BME (\%)

Mothers smoking at

$23.6(8.4) \quad 23.3(16.6-29.5) \quad 8.8,45.3$

$19.3(5.7) \quad 18.4(15.1-22.0) \quad 9.4,41.8$

$5.9(2.3) \quad 5.8(4.1-7.4) \quad 1.3,11.8$

$18.7(14.3) \quad 13.0(7.8-25.9) \quad 4.3,67.0$

$14.7(6.1) \quad 15.0(10.7-18.8) \quad 3.0,33.2$

\section{Non-London PCTs $(n=120)$}

Mean
(SD) Median (IQR)

minimum

$68.7(9.1) \quad 69.6(62.5-75.7) \quad 39.0,85.5$

39.7 (10.0) 39.9 (33.7-45.5) 19.2, 70.5

$29.2(7.4) \quad 29.3(24.2-33.2) \quad 14.3,58.2$

$23.1(8.3) \quad 22.8(16.4-28.5) \quad 8.8,45.3$

$17.8(4.4) \quad 17.6(14.5-21.2) \quad 9.4,32.3$

$6.6(1.9) \quad 6.3(5.3,7.9) \quad 2.8,11.8$

$13.1(8.7) \quad 10.9(7.3-16.9) \quad 4.3,67.0$

$16.7(4.8) \quad 16.5(13.7,-19.9) \quad 6.1,33.2$
London PCTS $(n=31)$

(SD) Median (IQR)

Minimum,

maximum

86.3 (6.6) $88.9(83.1-91.3) \quad 67.7,92.9$

67.5 (10.6) 71.3 (63.0-73.7) $38.1,83.1$

$41.2(8.8) \quad 42.5(35.0-48.6) \quad 20.5,57.5$

$25.6(8.8) \quad 25.0(16.7-31.9) \quad 10.1,41.8$

$25.2(6.4) \quad 24.5(20.1-30.4) \quad 15.4,41.8$

$3.1(1.1) \quad 2.8(2.2-3.8) \quad 1.3,5.6$

$40.4(10.9) \quad 42.9(33.9-47.5) \quad 16.4,61.9$

$6.6(2.9) \quad 5.9(4.4-7.5) \quad 3.0,13.6$

delivery (\%) $\ddagger$

${ }^{*}$ Restricted to 141 PCTs with data on breastfeeding at 6-8 weeks.

†A high score is indicative of greater deprivation.

†Restricted to 146 PCTs with data on smoking at delivery.

Information on maternal smoking at delivery was unavailable for a number of PCTs, leaving 144 and 137 PCTs included in the complete case analysis for breastfeeding initiation and

breastfeeding at 6-8 weeks (115 non-London PCTs and 29 London PCTs; 110 non-London PCTs and 27 London PCTs).

BME, Black and Minority Ethnic; IMD, Index of Multiple Deprivation; PCT, primary care trust. 


\section{PCTs outside London}

The relationship between any breastfeeding at $6-8$ weeks and each of the five sociodemographic variables under study is presented in a series of scatter plots in figure 1, with data points for non-London PCTs highlighted with solid markers. There are striking associations between breastfeeding and most of the sociodemographic variables: at the PCT level, the percentage of mothers breastfeeding tends to decrease as deprivation increases, and as the proportion of both younger mothers and maternal smoking increases. In general, breastfeeding rises in line with increases in the proportion of older mothers and the proportion of the population from a BME background. Scatter plots for breastfeeding initiation and exclusive breastfeeding at 6-8 weeks showed similar patterns (see online supplementary table S1).

ORs for the association between these sociodemographic factors and each of the three breastfeeding outcomes are shown in table 2. In a univariable analysis, breastfeeding (all outcomes) was significantly higher in those PCTs with a higher proportion of older mothers and a higher BME population. Lower breastfeeding at birth and at 6-8 weeks was observed in PCTs with increased deprivation and those areas with a higher prevalence of maternal smoking or teenage mothers.

In a multivariable analysis of non-London PCTs with complete data ( $\mathrm{n}=115$ for initiation, $\mathrm{n}=110$ for $6-8$ weeks), the following variables were independently associated with breastfeeding (all outcomes): lower area-based deprivation, more births to older women, and higher BME population (table 2). The proportions of teenage mothers and maternal smoking were no longer significant after adjustment for other variables ( $p$ values for teenage mothers in the last included model: 0.67, 0.49 and 0.39 for initiation, any and exclusive; $p$ values for maternal smoking in the last included model: $0.73,0.98$ and 0.63 for initiation, any and exclusive). The effect of deprivation was somewhat attenuated by adjustment for other factors, although when compared with the least deprived quintile, a significant decrease in odds was still observed in quintiles 4 and 5 for exclusive breastfeeding at $6-8$ weeks, and in quintile 5 for breastfeeding initiation and any breastfeeding at $6-8$ weeks. For breastfeeding initiation, the most deprived quintile (quintile 5) was associated with a reduction in the odds of $32 \%$ (adjusted OR 0.68 ) when compared with the least deprived quintile. Areas with higher proportions of older mothers, and increased BME population all had higher odds of breastfeeding at birth and at 6-8 weeks. Of these two factors, the strongest association was with older maternal age, where a unit increase in the percentage of mothers aged 35 or over was associated with a $6 \%$ increase in the odds of any breastfeeding at 6-8 weeks (adjusted OR 1.06) and a $5 \%$ increase in the odds of breastfeeding initiation or exclusive breastfeeding at 6-8 weeks (adjusted OR 1.05).

\section{London PCTs}

The same striking associations between breastfeeding and the sociodemographic variables are evident in the London PCTs (figure 1, highlighted with hollow markers). The only exception was area deprivation, which was not strongly associated with breastfeeding. These figures also provide strong evidence of the difference in both the sociodemographic and the breastfeeding profile of London PCTs compared with non-London PCTs.

A univariable analysis of the London PCTs showed significant associations between all breastfeeding outcomes and the maternal age profile of PCTs, the proportion of a PCT population from a BME background, and
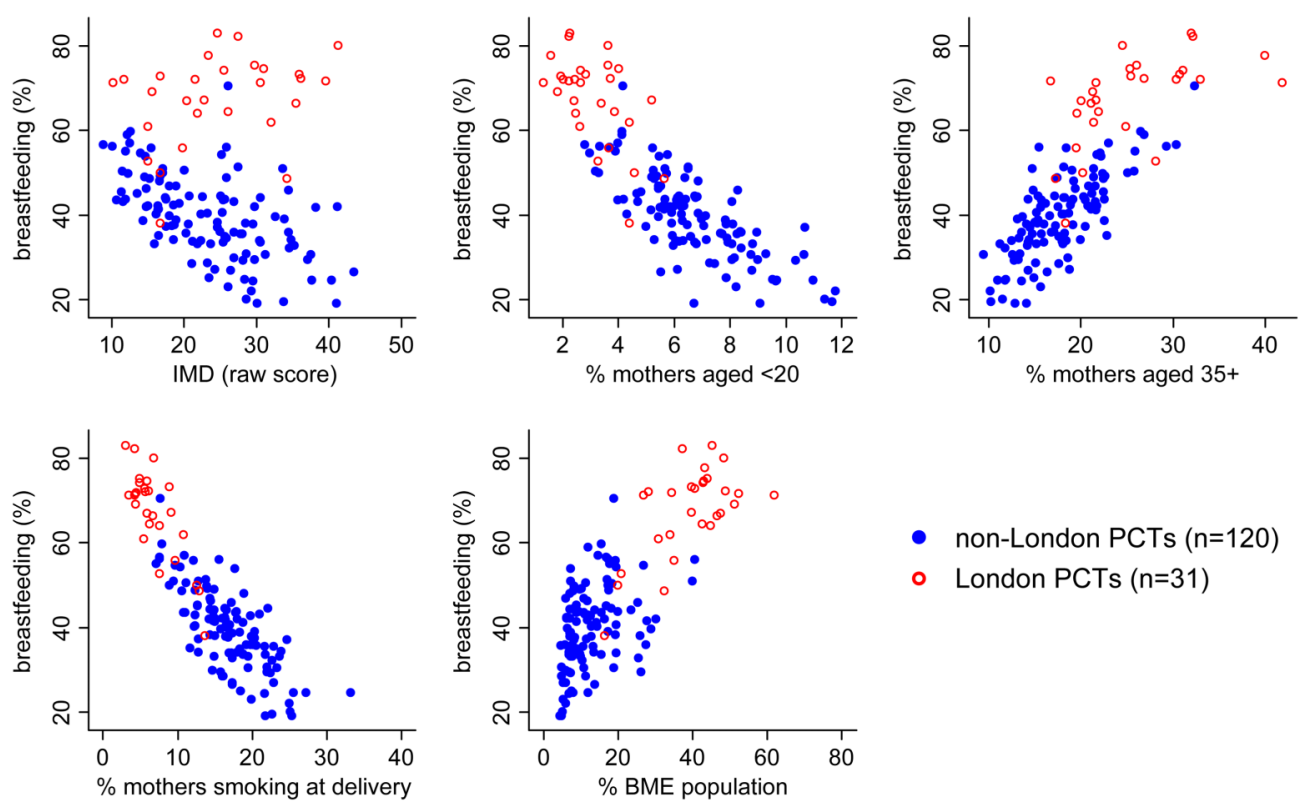

- non-London PCTs $(\mathrm{n}=120)$

- London PCTs $(n=31)$

Figure 1 Scatter plots for any breastfeeding at 6-8 weeks by sociodemographic factors (London vs non-London). IMD, Index of Multiple Deprivation (a high score is indicative of greater deprivation); BME, black and minority ethnic. 
Table 2 Association between the prevalence of breastfeeding and the prevalence of socio-demographic variables at the PCT level: non-London PCTs

\begin{tabular}{|c|c|c|c|c|c|c|c|c|c|c|c|c|}
\hline \multirow[b]{3}{*}{ Non-London PCTs } & \multicolumn{4}{|c|}{ Breastfeeding initiation $(n=115)$} & \multicolumn{4}{|c|}{ Any breastfeeding at $6-8$ weeks $(n=110)$} & \multicolumn{4}{|c|}{ Exclusive breastfeeding at $6-8$ weeks $(n=110)$} \\
\hline & \multicolumn{2}{|c|}{ Unadjusted } & \multicolumn{2}{|c|}{ Adjusted $^{*}$} & \multicolumn{2}{|c|}{ Unadjusted } & \multicolumn{2}{|c|}{ Adjusted† } & \multicolumn{2}{|c|}{ Unadjusted } & \multicolumn{2}{|c|}{ Adjusted¥ } \\
\hline & $\overline{\text { OR }}$ & $(95 \% \mathrm{Cl})^{-}$ & $\overline{\text { OR }}$ & $(95 \% \mathrm{Cl})$ & $\overline{\text { OR }}$ & $(95 \% \mathrm{Cl})$ & $\overline{\text { OR }}$ & $(95 \% \mathrm{Cl})$ & $\overline{\text { OR }}$ & $(95 \% \mathrm{Cl})$ & $\overline{\text { OR }}$ & $(95 \% \mathrm{Cl})$ \\
\hline \multicolumn{13}{|l|}{$\overline{I M D}$} \\
\hline $\begin{array}{l}\text { Quintile } 1 \text { (least } \\
\text { deprived) }\end{array}$ & 1.00 & & 1.00 & & 1.00 & & 1.00 & & 1.00 & & 1.00 & \\
\hline Quintile 2 & 0.82 & (0.68 to 1.00$)$ & 1.03 & (0.88 to 1.20$)$ & $0.74^{*}$ & $(0.60$ to 0.91$)$ & 0.98 & (0.85 to 1.12$)$ & $0.83^{*}$ & $(0.70$ to 0.98$)$ & 1.02 & (0.89 to 1.17$)$ \\
\hline Quintile 3 & $0.68^{\star \star \star}$ & (0.56 to 0.83 ) & 0.97 & (0.82 to 1.15$)$ & $0.61^{\star \star \star}$ & (0.49 to 0.75$)$ & 0.89 & (0.77 to 1.04$)$ & $0.67^{\star \star \star}$ & (0.56 to 0.79$)$ & 0.94 & (0.80 to 1.10$)$ \\
\hline Quintile 4 & $0.56^{\star * *}$ & (0.46 to 0.68$)$ & 0.84 & (0.70 to 1.01$)$ & $0.54^{\star \star \star}$ & (0.44 to 0.67$)$ & $0.83^{*}$ & (0.71 to 0.98$)$ & $0.61^{\star \star \star}$ & (0.51 to 0.72 ) & 0.90 & (0.76 to 1.06$)$ \\
\hline $\begin{array}{l}\text { Quintile } 5 \text { (most } \\
\text { deprived) }\end{array}$ & $0.50^{\star *}$ & (0.41 to 0.61$)$ & $0.68^{\star \star}$ & (0.54 to 0.86$)$ & $0.51^{\star \star \star}$ & (0.41 to 0.64$)$ & $0.69^{\star \star \star}$ & (0.57 to 0.85$)$ & $0.54^{\star \star \star}$ & (0.45 to 0.65$)$ & $0.79^{\star}$ & (0.64 to 0.97 ) \\
\hline Mothers aged $35+(\%)$ & $107^{\star \star \star}$ & (1.06 to 1.09$)$ & $1.05^{\star \star \star}$ & (1.04 to 1.07 ) & $1.08^{\star \star \star}$ & (1.06 to 1.09$)$ & $1.06^{\star \star}$ & (1.04 to 1.07 ) & $1.07^{\star \star \star}$ & (1.05 to 1.08$)$ & $1.05^{\star \star \star}$ & (1.04 to 1.07$)$ \\
\hline Mothers aged <20 (\%) & $0.86^{* * *}$ & (0.83 to 0.88$)$ & & & $0.84^{\star \star *}$ & (0.82 to 0.87$)$ & & & $0.88^{\star \star \star}$ & (0.86 to 0.90$)$ & & \\
\hline Population BME (\%) & $1.01^{*}$ & (1.00 to 1.02$)$ & $1.02^{\star \star \star}$ & (1.01 to 1.02 ) & $1.02^{\star \star *}$ & (1.01 to 1.03$)$ & $1.03^{* * *}$ & (1.02 to 1.04$)$ & $1.01^{\star}$ & (1.00 to 1.02$)$ & $1.01^{\star * *}$ & (1.01 to 1.02$)$ \\
\hline $\begin{array}{l}\text { Mothers smoking at } \\
\text { delivery }(\%)\end{array}$ & $0.94^{\star \star \star}$ & (0.93 to 0.95$)$ & & & $0.93^{\star \star \star}$ & (0.92 to 0.94$)$ & & & $0.95^{\star \star \star}$ & (0.94 to 0.96$)$ & & \\
\hline
\end{tabular}


Table 3 Association between the prevalence of breastfeeding and the prevalence of socio-demographic variables at the PCT level: London PCTs

\begin{tabular}{|c|c|c|c|c|c|c|c|c|c|c|c|c|}
\hline \multirow[b]{3}{*}{ London PCTS } & \multicolumn{4}{|c|}{ Breastfeeding initiation $(n=29)$} & \multicolumn{4}{|c|}{ Any breastfeeding at $6-8$ weeks $(n=27)$} & \multicolumn{4}{|c|}{$\begin{array}{l}\text { Exclusive breastfeeding at } 6-8 \text { weeks } \\
(n=27)\end{array}$} \\
\hline & \multicolumn{2}{|c|}{ Unadjusted } & \multicolumn{2}{|c|}{ Adjusted $^{*}$} & \multicolumn{2}{|c|}{ Unadjusted } & \multicolumn{2}{|c|}{ Adjusted $\dagger$} & \multicolumn{2}{|c|}{ Unadjusted } & \multicolumn{2}{|c|}{ Adjusted $\ddagger$} \\
\hline & $\overline{\text { OR }}$ & $(95 \% \mathrm{Cl})$ & $\overline{\text { OR }}$ & $(95 \% \mathrm{Cl})$ & $\overline{\text { OR }}$ & $(95 \% \mathrm{Cl})$ & $\overline{\text { OR }}$ & $(95 \% \mathrm{Cl})$ & $\overline{\text { OR }}$ & $(95 \% \mathrm{Cl})$ & $\overline{\text { OR }}$ & $(95 \% \mathrm{Cl})$ \\
\hline \multicolumn{13}{|l|}{$\overline{I M D}$} \\
\hline Quintile 1 (least deprived) & 1.00 & & 1.00 & & 1.00 & & 1.00 & & 1.00 & & 1.00 & \\
\hline Quintile 2 & 0.61 & $(0.35$ to 1.04$)$ & 1.31 & (0.94 to 1.84$)$ & 0.70 & $(0.43$ to 1.15$)$ & & & $0.63^{*}$ & (0.35 to 0.93$)$ & & \\
\hline Quintile 3 & 1.22 & $(0.71$ to 2.10$)$ & $1.38^{*}$ & (1.03 to 1.85$)$ & 1.39 & (0.85 to 2.28$)$ & & & 1.07 & $(0.72$ to 1.57$)$ & & \\
\hline Quintile 4 & 1.34 & (0.75 to 2.37$)$ & $1.46^{*}$ & (1.06 to 2.00$)$ & 1.48 & (0.88 to 2.50$)$ & & & 0.95 & (0.62 to 1.43 ) & & \\
\hline Quintile 5 (most deprived) & 1.10 & (0.69 to 1.75$)$ & $1.71^{\star \star *}$ & (1.30 to 2.25$)$ & 1.18 & $(0.76$ to 1.84$)$ & & & 0.82 & $(0.58$ to 1.17$)$ & & \\
\hline Mothers aged $35+(\%)$ & $1.05^{\star \star *}$ & (1.03 to 1.08$)$ & $1.04^{* * *}$ & (1.02 to 1.06$)$ & $1.04^{\star *}$ & (1.02 to 11.07$)$ & $1.06^{* * *}$ & (1.04 to 1.07$)$ & $1.05^{\star * *}$ & (1.03 to 1.06$)$ & $1.06^{\star * *}$ & (1.04 to 1.07$)$ \\
\hline Mothers aged <20 (\%) & $0.79^{\star \star}$ & (0.68 to 0.92$)$ & & & $0.79^{\star \star}$ & (0.69 to 0.91$)$ & & & $0.82^{\star * *}$ & (0.74 to 0.91$)$ & & \\
\hline Population BME (\%) & $1.02^{*}$ & (1.00 to 1.03$)$ & & & $1.03^{\star \star *}$ & (1.01 to 1.04$)$ & $1.03^{\star \star *}$ & (1.03 to 1.04$)$ & 1.01 & (1.00 to 1.02$)$ & & \\
\hline $\begin{array}{l}\text { Mothers smoking at delivery } \\
(\%)\end{array}$ & $0.88^{\star * \star}$ & (0.84 to 0.91$)$ & $0.90^{\star * *}$ & (0.87 to 0.94$)$ & $0.88^{* * *}$ & (0.84 to 0.91$)$ & & & $0.92^{\star * *}$ & (0.89 to 0.95$)$ & $0.95^{\star *}$ & (0.92 to 0.98$)$ \\
\hline \multicolumn{13}{|c|}{$\begin{array}{l}{ }^{*} p<0.05 ;{ }^{* *} p<0.01 ;{ }^{* * *} p<0.001 . \\
\text { *Variables in model: IMD, percentage of births to older mothers, percentage of mothers smoking at delivery. } \\
\text { †Variables in model: percentage of births to older mothers, percentage of population BME. } \\
\text { fVariables in model: percentage of births to older mothers, percentage of mothers smoking at delivery. } \\
\text { BME, Black and Minority Ethnic; IMD, Index of Multiple Deprivation; PCT, primary care trust. }\end{array}$} \\
\hline
\end{tabular}



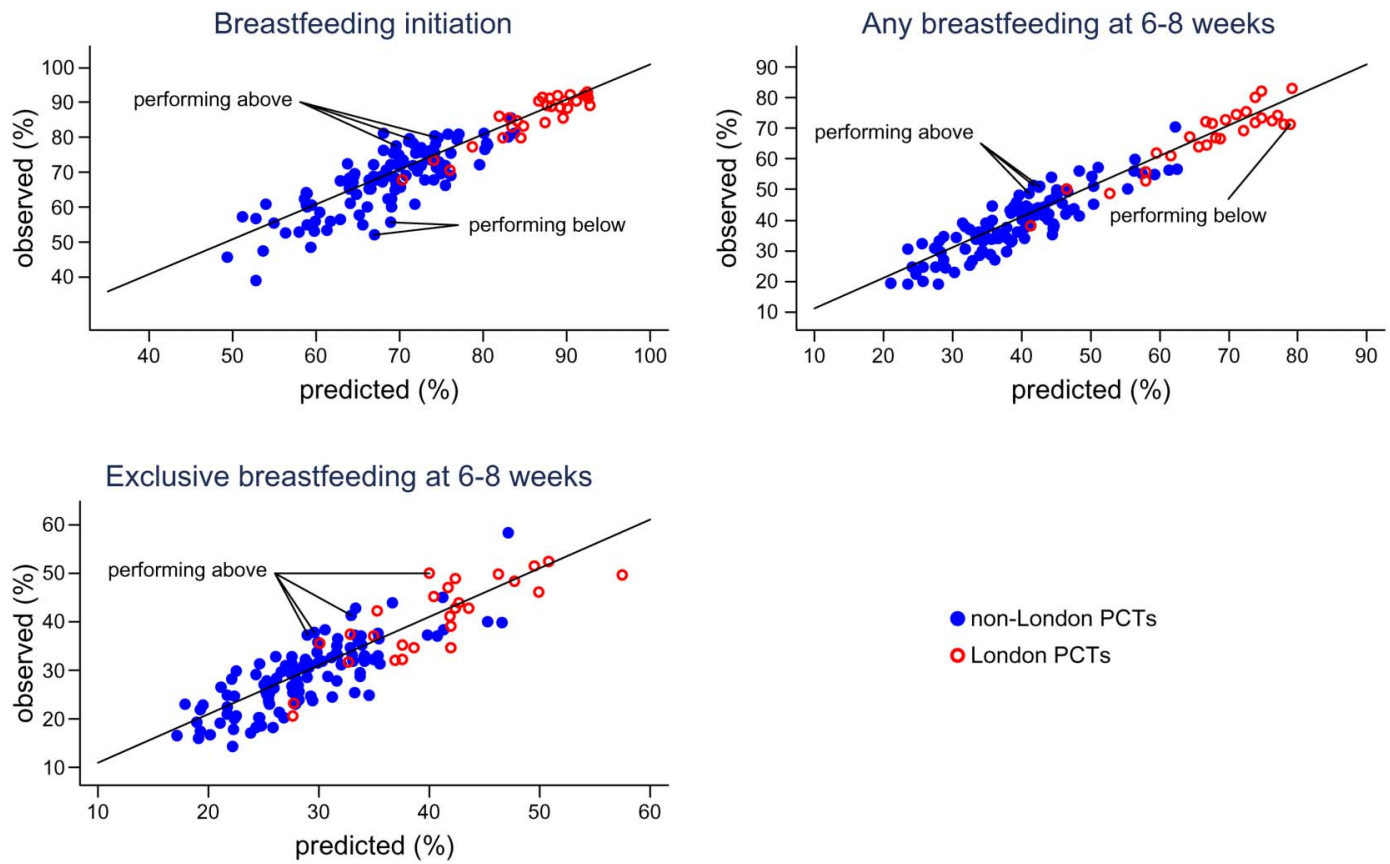

Figure 2 Observed versus predicted proportions of any breastfeeding at 6-8 weeks.

NB, In some cases PCTs classified as potential outliers reported similar breastfeeding figures to PCTs not identified as potential outliers, this discrepancy is due to differences in PCT size.

Breastfeeding initiation: performing above - Hampshire, Sheffield, Somerset; performing below - Dudley, Sefton. Any breastfeeding at 6-8 weeks: performing above - Devon, Leeds, Sheffield; performing below - Brent. Exclusive breastfeeding at 6-8 weeks: performing above - City and Hackney, Devon, Leeds, Sheffield.

maternal smoking (table 3). Area-based deprivation showed no or little significant association with breastfeeding in London.

In the multivariable analysis, factors independently associated with breastfeeding initiation were area deprivation, older maternal age and maternal smoking (table 3). The proportion of teenage mothers and BME population were not retained in the final model as they were no longer significant after adjustment ( $p$ values for the last model including these variables: 0.73 for teenage mothers, 0.94 for BME). Increased maternal smoking at delivery was associated with lower breastfeeding initiation, and in line with the results for PCTs outside London, increased prevalence of older mothers was associated with higher breastfeeding initiation. However, contrary to the results observed outside London, increased deprivation appeared to be independently associated with higher breastfeeding initiation. Quintiles 3-5 had a significantly increased OR compared with the least deprived quintile 1 ; for quintile 5 (most deprived PCTs), the adjusted OR was 1.71.

After adjustment for other factors, deprivation was not independently associated with breastfeeding at 6-8 weeks in London PCTs (not taken forward to multivariable modelling in analysis of any breastfeeding; $p$ values for the last model including this variable in the analysis of exclusive breastfeeding ranged from 0.21 to 0.74 ). Older maternal age was associated with breastfeeding at 6-8 weeks, with an OR of 1.06 for both any and exclusive breastfeeding. This is equivalent to a $6 \%$ increase in the odds of breastfeeding for every $1 \%$ increase in the proportion of older mothers. The proportion of teenage mothers was not associated with either any breastfeeding or exclusive breastfeeding at 6-8 weeks after adjustment for other factors ( $p$ values for the last models including this variable: 0.28 for any breastfeeding, 0.74 for exclusive breastfeeding). The proportion of the local population from a BME background was independently associated with any breastfeeding at 6-8 weeks (OR 1.03/unit change), and maternal smoking associated with decreased exclusive breastfeeding. In the final multivariable model for any breastfeeding at 6-8 weeks, maternal smoking did not retain significance ( $p$ value for the last model including this variable 0.58$)$.

\section{Observed and predicted proportions}

Figure 2 shows the breastfeeding proportions observed in each PCT plotted against the breastfeeding proportion that would be predicted based on the multivariable models shown in tables 2 and 3. The vast majority of PCTs reported proportions consistent (within two standardised residuals) with the proportions predicted by the models. Three PCTs (all non-London) reported breastfeeding initiation as being considerably higher than predicted, and two PCTs (both non-London) reported figures lower than predicted (outliers are highlighted in figure 2). Three PCTs (all non-London) and four PCTs (three non-London, one London) reported proportions of any and exclusive breastfeeding higher than predicted. One London PCT reported the proportion of any breastfeeding at 6-8 weeks as lower than 
expected. Online supplementary table S2 shows the observed and predicted breastfeeding proportions for each PCT.

\section{DISCUSSION}

To our knowledge, this is the first UK study designed to investigate the relationship between sociodemographic factors and breastfeeding at the area level, an analysis which is important, given that services are commissioned and delivered at this level. There was enormous variation in the area-based rates of breastfeeding. However, after adjusting for sociodemographic factors, most areas have breastfeeding rates within the expected range of the national average, albeit a relatively low national average (eg, $45 \%$ any breastfeeding at 6-8 weeks). The areabased analysis revealed some striking associations between sociodemographic factors and breastfeeding; these persisted after adjustment for other factors. For example, an increase in the proportion of mothers aged 35 or older from $15 \%$ to $20 \%$ is associated with a $30 \%$ increase in the odds for area-level any breastfeeding at 6-8 weeks.

\section{Limitations}

This study used aggregate data and as such is subject to the usual limitation that causality cannot be inferred. Although the use of routine data has many benefits, for example, wide geographical coverage, there are also inherent disadvantages such as the difficulty in assessing data quality. It is also possible that higher levels of breastfeeding can be partially explained by area-level factors not measured in this study, for example, a greater number of accessible breastfeeding services. We included all socioeconomic indicators routinely available at the PCT level. There may be other relevant factors that would have been useful to include, such as levels of maternal education.

DH for England does not make raw figures for annual outturn breastfeeding data routinely available. In order to model figures in our regression analysis, we relied on quarterly actual data. These data may differ very slightly from annual outturn data, but there is no reason to suspect that trends would be different. One advantage of our method (summing breastfeeding data across quarters) was that we were able to include PCTs with one or two data quarters missing, thus minimising data loss. However, a small number of PCTs were excluded as breastfeeding data did not meet our stipulated criteria (acceptable data quality for at least two quarters). In addition, the small number of PCTs in London (27-29 depending on analysis) may affect our confidence in the results from our analysis of London PCTs: it is unclear whether lack of association reflects a true lack of effort or is simply the consequence of an underpowered analysis. The observed breastfeeding proportions used in this analysis are likely to be an underestimate of the true number breastfeeding as we mirrored the denominators used by
$\mathrm{DH}$, which assumes that those for whom a breastfeeding status was not recorded are not breastfeeding.

Data on smoking at delivery were not available for all PCTs. Six PCTs were not eligible for inclusion as reported data did not meet DH quality checks. Despite this, smoking status was available for $99 \%$ of maternities in 2010-2011 (range for individual PCTs included in this analysis $95.5-100 \%)$.

Our ethnicity indicator related to the general PCT population rather than the maternal population. Given the high level of missing data on maternal ethnicity from HES (approximately $8 \%$ in 2009-2010 ${ }^{34}$ ), using these data would have resulted in a reduction in our sample size. We compared the general ethnicity data with the HES maternal ethnicity data and noted that it correlated well, although the HES maternal ethnicity data reported higher proportions across all PCTs, probably due to the younger age profile of BME populations. We combined all non-White groups into a single BME indicator. This helped to minimise potential problems due to the small numbers of certain ethnic groups in many PCTs. This decision was also supported by strong evidence that all non-White women are more likely to initiate and continue breastfeeding when compared with White women. ${ }^{11}$ However, our approach left us unable to examine the separate contribution of individual ethnic groups or relevant factors such as migration history or acculturation status ${ }^{35}$ to breastfeeding rates.

\section{Interpretation of results}

There was compelling evidence of a strong area effect of older maternal age on breastfeeding, with a $1 \%$ increase in the percentage of older mothers in a PCT associated with a $4-6 \%$ increase in the odds of breastfeeding. This trend was consistent across all outcomes and in both London and non-London PCTs, and is in line with evidence from individual level studies that older mothers are more likely to initiate and continue breastfeeding. ${ }^{11} 24$

Outside London, the proportion of the PCT population from a BME background was associated with breastfeeding, with a unit increase in BME population resulting in a $1-3 \%$ increase in the odds of breastfeeding. Non-white ethnicity has consistently been linked to increased breastfeeding in individual level studies, ${ }^{16} 172022{ }^{26}$ although there is some variation between individual ethnic groups and by acculturation status. ${ }^{35}$ The existing literature suggests that the strongest overall effect of ethnicity is on initiation and continuation, with minimal differences by ethnicity in the number of women who breastfeed exclusively. ${ }^{36}{ }^{37}$ Although the proportion of the PCT population from a BME background was associated with all breastfeeding outcomes outside London, it was only independently associated with breastfeeding at 6-8 weeks in London. The fact that we did not identify an independent effect of BME population on initiation in London may be partly due to the high rate of BME in the London PCTs (mean value $40 \%$ ) making it difficult to detect an independent 
effect of ethnicity. In addition, it may be that the high rates of breastfeeding in ethnically diverse areas supports the concept of 'community ethnicity', ${ }^{20}$ whereby some groups of White women appear to be more likely to breastfeed if they reside in an area with a high BME population. Differences in the composition of BME populations in London compared with outside London may also help to explain the inconsistency in the observed effect of BME on breastfeeding.

PCTs in the most deprived quintile had a $21-32 \%$ reduced odds of breastfeeding compared with PCTs in the least deprived quintile. In London PCTs, results were less consistent after adjustment, with area deprivation only associated with breastfeeding initiation, and this association being in the opposite direction to that observed outside London (increased deprivation associated with increased odds of initiation). This perhaps highlights the complex relationships between ethnicity, socioeconomic status and breastfeeding behaviour. BME populations tend to cluster in more deprived neighbourhoods. Women from non-White backgrounds are more likely to breastfeed. In general, mothers from lower socioeconomic backgrounds are less likely to breastfeed. There is evidence that this latter trend cannot be generalised to mothers from non-White backgrounds. ${ }^{17}$ Several studies have found that the effects of deprivation, ${ }^{17}$ socioeconomic status ${ }^{19}$ and income ${ }^{22}$ are negligible when looking at breastfeeding among certain minority ethnic groups. Outside London, both deprivation and area level ethnicity remained independent predictors of breastfeeding even after adjusting for the other. Within London, the effect of one appeared to be attenuated by the other, except when looking at exclusive breastfeeding, which was not independently associated with either.

Our analysis was designed to explain the variation in breastfeeding between PCTs. Only a handful of PCTs reported breastfeeding figures substantially above or below the proportions predicted by our models. The majority of outliers were PCTs with observed proportions higher than expected based on the national average having adjusted for sociodemographic factors, though two PCTs did report breastfeeding initiation as being lower than predicted and one PCT had a lower than expected proportion of any breastfeeding at $6-8$ weeks.

\section{Implications}

Our results demonstrate that while the area-based breastfeeding rates vary enormously, much of this variation is explained by the sociodemographic profile of the area. Currently, breastfeeding data provided at the PCT level for comparative purposes are unadjusted ${ }^{38}$ and may result in a misleading assessment of local performance. Adjusted breastfeeding figures as reported in this study may be used to identify areas with higher or lower than expected rates of breastfeeding. For those performing above expected levels, there may be lessons to be learnt from examining local service provision.
The sociodemographic context within which a breastfeeding service is implemented or evaluated is clearly important. ${ }^{39} \mathrm{~A}$ 'one size fits all' approach to breastfeeding support is unlikely to demonstrate a strong effect at the population level over and above the 'background noise' of such strong sociodemographic effects. Interventions which are tailored to the needs of a particular setting are more likely to be effective, ${ }^{40}$ particularly those that follow local needs assessment. Our findings can be used to help inform the primary focus of an intervention, for example, whether the emphasis should be on breastfeeding initiation, duration or exclusivity or a combination of these outcomes. ${ }^{40}$ The size of effects observed in our study may also inform estimates of the likely effects of breastfeeding interventions in a trial or other setting. In situations where the required trial size is too large to be feasible, other forms of evaluation, such as case studies of high performing PCTs, are likely to be a more suitable approach.

In the new (post-April 2013) National Health Service structure in England, it is uncertain which organisations will be responsible for commissioning breastfeeding services. However, our results will be relevant to whichever local structures take over this function, particularly given that many of the geographical areas presented here will be recognisable in the new structure. Although most PCTs are performing at the level expected given the current trends, the overall breastfeeding rates are still low and fall short of the UK recommendations for mothers to breastfeed exclusively for the first 6 months of life.

\section{CONCLUSION}

Our results confirm the importance of sociodemographic indicators of breastfeeding, and provide evidence that these indicators explain much of the heterogeneity between PCTs in terms of the proportion of mothers breastfeeding. However, there is little room for complacency; while some areas in England now have high rates of breastfeeding initiation, almost all have low rates of continuation, particularly of exclusive breastfeeding. In order to maximise the likelihood of success, interventions designed to increase breastfeeding at the area level will need to be tailored to the sociodemographic context, and monitoring and assessment of areabased rates will need to take these factors into account.

Contributors MAQ and JJK conceived the study and all authors contributed to the design of the study. MAQ and LLO designed and conducted the analysis. All authors were involved in the interpretation of the data. LLO and $M A Q$ wrote the initial draft of the manuscript, and all authors contributed to revising consecutive drafts. All authors approved the final version of the manuscript. All authors had full access to all the data in the study and can take responsibility for the integrity of the data and the accuracy of the data analysis. MAQ and LLO are the guarantors for the study.

Funding This paper reports on an independent study which is funded by the Policy Research Programme in the Department of Health (Policy Research Unit in Maternal Health and Care, reference no. 108/0001). The views expressed are not necessarily those of the department. The funder had no 
role in: the design and conduct of the study; the analysis and interpretation of the data, as well as the drafting of the paper and decision to submit the paper for publication.

Competing interests None.

Provenance and peer review Not commissioned; externally peer reviewed.

Data sharing statement Observed and predicted breastfeeding rates by PCT are provided as Supplementary Data. For further information please contact the corresponding author.

\section{REFERENCES}

1. Ip S, Chung M, Raman G, et al. Breastfeeding and maternal and infant health outcomes in developed countries. Rockville, MD: Agency for Healthcare Research and Quality, 2007. Evidence report/ technology assessment 2009;153.

2. Renfrew MJ, Pokhrel S, Quigley M, et al. Preventing disease and saving resources: the potential contribution of increasing breastfeeding rates in the UK. London: UNICEF, 2012.

3. Quigley MA, Cumberland P, Cowden JM, et al. How protective is breast feeding against diarrhoeal disease in infants in 1990s England? A case-control study. Arch Dis Child 2006;91:245-50.

4. Quigley MA, Kelly YJ, Sacker A. Breastfeeding and hospitalization for diarrheal and respiratory infection in the United Kingdom Millennium Cohort Study. Pediatrics 2007;119:e837-42.

5. Fisk CM, Crozier SR, Inskip HM, et al. Breastfeeding and reported morbidity during infancy: findings from the Southampton Women's Survey. Matern Child Nutr 2011;7:61-70.

6. Henderson G, Craig S, Brocklehurst $\mathrm{P}$, et al. Enteral feeding regimens and necrotising enterocolitis in preterm infants: a multicentre case-control study. Arch Dise Childhood 2009;94: F120-F23.

7. Collaborative Group on Hormonal Factors in Breast Cancer Möller T, Olsson H, Ranstam J, et al. Breast cancer and breastfeeding: collaborative reanalysis of individual data from 47 epidemiological studies in 30 countries, including 50,302 women with breast cancer and 96,973 women without the disease. Lancet 2002;360:187-95.

8. Horta BL, Bahl B, Martines JC, et al. Evidence on the long-term effects of breastfeeding. Geneva: WHO, 2007.

9. Quigley MA, Hockley C, Carson C, et al. Breastfeeding is associated with improved child cognitive development: a population-based cohort study. J Pediatr 2012;160:25-32.

10. Scientific Advisory Committee on Nutrition (SACN), Subgroup on Maternal and Child Nutrition (SMCN). Paper for discussion: introduction of solid foods, agenda item: 3. 2003. SMCN/03/08., 2008.

11. McAndrew F, Thompson J, Fellows L, et al. (2012). Infant feeding survey 2010. Leeds: Health and Social Care Information Centre, 2012.

12. Cattaneo A, Burmaz $\mathrm{T}$, Arendt $\mathrm{M}$, et al. Protection, promotion and support of breast-feeding in Europe: progress from 2002 to 2007. Public Health Nutr 2010;13:751-9.

13. Department of Health. Improvement, expansion, reform - the next three year: priorities and planning framework 2003-2006. London: Department of Health, 2002.

14. HM Government. PSA Delivery Agreement 12: improve the health and wellbeing of children and young people. London: HMSO, 2007.

15. Department of Health. Improving outcomes and supporting transparency. Part 1: a public health outcomes framework for England, 2013-2016. London: Department of Health, 2012.

16. Agboado G, Michel E, Jackson E, et al. Factors associated with breastfeeding cessation in nursing mothers in a peer support programme in Eastern Lancashire. BMC Pediatr 2010;10:3

17. Baker D, Garrow A, Shiels C. Inequalities in immunisation and breast feeding in an ethnically diverse urban area: cross-sectional study in Manchester, UK. J Epidemiol Community Health 2011;65:346-52.
18. Dennis CL. Breastfeeding initiation and duration: a 19902000 literature review. J Obstetr Gynecol Neonatal Nurs 2002;31:12-32.

19. Griffiths LJ, Tate AR. Do early infant feeding practices vary by maternal ethnic group? Public Health Nutr 2007;10:957-64.

20. Griffiths LJ, Tate AR, Dezateux C, Group tMCSCH. The contribution of parental and community ethnicity to breastfeeding practices: evidence from the Millennium Cohort Study. Int $J$ Epidemiol 2005;34:1378-86.

21. Kelly $\mathrm{Y}$, Watt $\mathrm{R}$. Breast-feeding initiation and exclusive duration at 6 months by social class-results from the Millennium Cohort Study. Public Health Nutr 2005;8:417-21.

22. Kelly YJ, Watt RG, Nazroo JY. Racial/ethnic differences in breastfeeding initiation and continuation in the United Kingdom and comparison with findings in the United States. Pediatrics 2006;118 e1428-35.

23. Matijasevich A, Victora CG, Lawlor DA, et al. Association of socioeconomic position with maternal pregnancy and infant health outcomes in birth cohort studies from Brazil and the UK. J Epidemiol Community Health 2012;66:127-35.

24. Mclnnes R, Love J, Stone D. Independent predictors of breastfeeding intention in a disadvantaged population of pregnant women. BMC Public Health 2001;1:10.

25. Brown AE, Raynor P, Benton D, et al. Indices of Multiple Deprivation predict breastfeeding duration in England and Wales. Eur J Public Health 2010;20:231-5.

26. McMillan $\mathrm{B}$, Conner M, Woolridge $\mathrm{M}$, et al. Predicting breastfeeding in women living in areas of economic hardship: explanatory role of the theory of planned behaviour. Psychol Health 2008;23:767-88.

27. Skafida V. The relative importance of social class and maternal education for breast-feeding initiation. Public Health Nutr 2009;12:2285-92.

28. Turcksin R, Bel S, Galjaard S, et al. Maternal obesity and breastfeeding intention, initiation, intensity and duration: a systematic review. Matern Child Nutr 2012: doi: 10.1111/j.1740-8709.2012. 00439.x

29. Freemantle N, Wood J, Griffin C, et al. What factors predict differences in infant and perinatal mortality in primary care trusts in England? A prognostic model. BMJ 2009;339:2892.

30. Department of Health. Statistical releases on breastfeeding, smoking and obesity: Department of Health, 2012.

31. McLennan D, Barnes $\mathrm{H}$, Noble $\mathrm{M}$, et al. The English indices of deprivation 2010. London: Department for Communities and Local Government, 2011.

32. Office of National Statistics. Population estimates for ethnic group (experimental) Mid-2009, 2011.

33. Child and Maternal Health Observatory. Child and Maternal Health Observatory (ChiMat), http://atlas.chimat.org.uk/IAS/advanceddataviews/ (accessed Nov 2012).

34. Health and Social Care Information Centre. Hospital Episode Statistics: How good is HES ethnic coding and where do the problems lie? (Revised for the 2009-2010 HES Data Year). Leeds: Health and Social Care Information Centre, 2011.

35. Hawkins SS, Lamb K, Cole TJ, et al. Influence of moving to the UK on maternal health behaviours: prospective cohort study. BMJ 2008;336:1052-5

36. Bolling K, Grant C, Hamlyn B, et al. Infant feeding survey 2005. London: The Information Centre for Health and Social Care, 2007.

37. Thomas M, Avery V. Infant feeding in Asian families: early feeding practices and growth. London: The Stationery Office, 1997.

38. RightCare. NHS Atlas of Variation: Child Health (Online InstantAtlas Version), http://www.sepho.org.uk/extras/maps/NHSatlasChildHealth/ atlas.html (accessed Nov 2012).

39. Hoddinott P, Seyara R, Marais D. Global evidence synthesis and UK idiosyncrasy: why have recent UK trials had no significant effects on breastfeeding rates? Matern Child Nutr 2011;7:221-7.

40. Dyson L, Renfrew MJ, McFadden A, et al. Policy and public health recommendations to promote the initiation and duration of breast-feeding in developed country settings. Public Health Nutr 2010;13:137-44. 